\title{
MASTOID ABSCESS: A RARE PRESENTATION OF EXTERNAL AUDITORY CANAL CARCINOMA
}

Tarun Sood ${ }^{1}$, Kaustubh Kahane ${ }^{2}$, Col S. K. Singh ${ }^{3}$, Suresh Mokamati ${ }^{4}$

\section{HOW TO CITE THIS ARTICLE:}

Tarun Sood, Kaustubh Kahane, Col S. K. Singh, Suresh Mokamati. "Mastoid Abscess: A Rare Presentation of External Auditory Canal Carcinoma". Journal of Evolution of Medical and Dental Sciences 2015; Vol. 4, Issue 40, May 18; Page: 7074-7078, DOI: 10.14260/jemds/2015/1028

\begin{abstract}
Squamous cell carcinoma (SCC) of the temporal bone and external auditory canal is a very rare tumor with a reported incidence of between 1 to 6 cases per million per year. SCC of the temporal bone and external auditory canal is very rare, an adequate tumor staging system and treatment has been difficult to develop because of limited number of cases reporting to any clinical centre. Usual presentation of a carcinoma of temporal bone is in the form of blood stained ear discharge with associated cranial nerve palsies. In this paper, we present a case of squamous cell carcinoma of the external auditory canal, who presented with an unusual presentation of a mastoid abscess which led to a diagnostic dilemma. However due to advanced age of presentation and short history, clinical suspicion of associated malignancy was made. Abscess was drained and biopsy from external auditory canal taken for histopathological analysis which confirmed it to be a well differentiated SCC.
\end{abstract}

KEYWORDS: Squamous cell carcinoma, external auditory canal, temporal bone.

INTRODUCTION: Carcinoma arising in the external auditory canal and temporal bone is very rare and comprises $<0.2 \%$ of head and neck neoplasms with an age adjusted incidence of $1 / 1,000,000$ per year in women and 0.8/1,000,000 in men.[1] However, squamous cell carcinoma (SCC) is the most common neoplasm, which accounts for 0-90percent of neoplasms of the temporal bone.[2] This is an aggressive disease with prognosis dependent on the stage of disease and the primary treatment. Other primary histological types of neoplasms arising in this area include adenocarcinoma, adenoid cystic carcinoma, mucoepidermoid carcinoma, basal cell carcinoma, ceruminous carcinoma, and rhabdomyosarcoma. Chronic otitis media ( $\mathrm{COM})$ and radiation to the temporal bone are debatable risk factors. ${ }^{[3,4]}$ Clinical history is of paramount importance if SCC of the temporal bone is to be diagnosed early. Many patients have associated long standing ear discharge, change in its character may be the only presenting symptom of this grave pathology. Patients may also present with cranial nerve palsies which further leads to diagnostic dilemma as to whether cranial nerve palsy is because of complications of COM or asoosciated SCC or malignant otitis externa. ${ }^{[5]}$ No literature could be found linking mastoid abscess as the presentation of external auditory canal. The following case of SCC external auditory canal did exactly the same and presented as a case of mastoid abscess thereby we felt the need to report the case.

CASE: A 53-year-old male presented with a history of swelling and pain over right zygomatic and mastoid region of 7 days duration [Fig. 1]. He also gave history of foul smelling, scanty and occasionally blood stained ear discharge from right ear of 15 days duration. There was associated history of hearing loss and otalgia of same duration. There was no history of fever, facial weakness, headache, vomiting or vertigo. On Examination it was a $6 \times 5 \mathrm{~cm}$ swelling in Right Zygomatic region 


\section{CASE REPORT}

extending to mastoid region which was erythematous, tender, soft with rise of local temperature. The EAC was filled with granulations which were bleeding on touch.

The tympanic membrane could not be visualized. A provisional diagnosis of COM with complications in the form of mastoid abscess was made and the patient was taken for an Incision \& Drainage (I\&D) and pus was drained. However suspicion of underlying malignancy was made in view of short history of ear discharge. Therefore, biopsy from EAC was taken in the same sitting and sent for HPE. Further management posed difference in opinion amongst senior surgeons. One view was to take up the patient for urgent modified radical mastoidectomy to prevent recollection of pus and other was to wait for the biopsy report.

Urgent HRCT temporal bone and CECT brain and neck was sought with an intention to rule out underlying intracranial complication and delineate the extent of disease. Meanwhile patient also developed House Brackman grade III facial nerve palsy which further necessitated surgical intervention to clear the underlying pathology in case it was COM. Meanwhile CT scan report and images were received which revealed irregular heterogenoulsy enhancing lesion in temporozygomatic region measuring $7.6 \times 5.1 \times 8.3 \mathrm{~cm}$ likely originating from Right EAC which was abutting the Temporal Lobe, eroding cochlea, semicircular circular canals and squamous part of temporal bone with filling defect in ipsilateral sigmoid sinus closing abutting Internal carotid artery [Fig. 2]. There was also an enlarged lymph node of $17 \mathrm{~mm}$ on Right level II. With these findings plan for surgical intervention was withheld and decision was taken to wait for biopsy report and then intervene if required. The biopsy report was suggestive of a keratinizing well differntiated Squamous cell Carcinoma. Therefore, with this diagnosis and other supporting investigations, we came to a diagnosis of SCC of temporal bone with staging of cT4N1M0. In view of advanced stage of presentation. The patient was planned for curative radiotherapy.

DISCUSSION: Squamous cell carcinoma of the external auditory meatus, middle ear and temporal bone is an unusual and rare malignancy, with not much evidence to support available treatment options which also explains the fact that there is no American Joint Committee on Cancer (AJCC) or Union for International Cancer Control (UICC) staging system for this unusual site of malignancy.[6] The University of Pittsburgh staging system for primary squamous cell cancer of the external auditory canal was proposed by Arriaga et al. in 1990 to attempt to classify the disease prior to the treatment.[7] The diagnosis of squamous cell carcinoma of the temporal bone and external auditory canal is based on histopathological examination of tissue of the tumor from the ear.

Most of the Squamous cell Carcinomas of the external auditory canal occur in the fifth and the sixth decade of life. Symptomatology of Squamous cell carcinoma External ear canal as per,(8) is most commonly ear discharge in almost all cases, otalgia in 74 percent of cases, hearing loss in $62 \%$, bleeding in $28 \%$, facial weakness in $12 \%$ and vertigo in $10 \%$ of cases. However, the patient reported above presented with mastoid abscess, this association could not be found in literature after exhaustive search. The disease often creates a diagnostic dilemma due to similar presentation as a otitis media. Squamous cell carcinoma of the external auditory canal presenting as a zygomatic abscess is a rare entity altogether.

The patient in the above mentioned case depicted a picture of Mastoid abscess probably as a result of Chronic otitis media, squamosal type. The short duration of the ear discharge was the only factor which led us to a decision to take a simultaneous biopsy before proceeding with definitive management of Chronic otitis media, diagnosis of which however is always clinical. However, issue 
before the team was to whether take the patient for definitive surgery for chronic otitis media or wait for HPE report, which would entail delay in surgery and could have had detrimental effects in the form of extension of disease to other complications like facial nerve palsy, meningitis and brain abscess. To assist in decision making and to have more information about the nature and extent of disease in addition to ruling out intracranial complications HRCT temporal bone, CECT brain was done which showed extensive bony destruction with intracranial extension. With imaging findings suspicion of malignancy superseded that of chronic otitis media. Hence decision was taken to wait for histopathology report and proceed which indeed revealed squamous cell carcinoma.

The treatment of squamous cell cancer of the external auditory canal depends on the staging of the tumor which includes lymph nodes metastasis and the facial nerve involvement during presentation. The preferred treatment often consists of a combination of en bloc surgical resection of the primary tumor with tumor-free surgical margins followed by postoperative chemotherapy and radiotherapy. The surgery that is often performed is the lateral temporal bone resection (LTBR) or a subtotal temporal bone resection (STBR) or a Total temporal bone resection.[7] Considering the extent of disease in this case, wide surgical excision of tumour was not possible due to involvement of carotid canal and intracerebral invasion. The patient was thereby planned for a curative radiotherapy.

Therefore to conclude Squamous cell carcinoma of the external ear canal and temporal canal is very rare malignancy of head and neck and its presentation as mastoid abscess further rare. It is very likely to be misdiagnosed as chronic otitis media and high index of suspicion is the key to diagnosis. In cognizance to principles of all malignancies, early detection of the tumor before extensive allows for better treatment and better prognosis.[9] The purpose of publishing this case was the unusual presentation and the diagnostic dilemma that was created due to the presentation. T4 tumors have a very poor prognosis and the survival rate too is very low.

\section{REFERENCES:}

1. Morton RP, Stell PM, Derrick PP. Epidemiology of cancer of 29. Moody SA, Hirsch BE, Myers EN. Squamous cell carcinoma the middle ear cleft. Cancer. 1984; 53: 1612-7.

2. National Cancer Institute, "Cancer facts. Head and neck cancer: questions and answers," http: //www.cancer.gov/cancertopics/factsheet/Sites-Types/head-and-neck/.

3. Moffat DA, Grey P, Ballagh RH, Hardy DG. Extended 28. Grandis JR, Hirsch BE, Yu VL. Simultaneous presentation of temporal bone resection for squamous cell carcinoma. malignant external otitis and temporal bone cancer. Otolaryngology and Head and Neck Surgery. 1997; 116: Archives of Otolaryngology - Head and Neck Surgery.617-23.

4. Goh YH, Chong VF, Low WK. Temporal bone tumours in patients irradiated for nasopharyngeal neoplasm. Journal 37. Hirsch BE. Staging system revision. Archives of

a. of Laryngology and Otology. 1999; 113: 222-8.

5. Grandis JR, Hirsch BE, Yu VL. Simultaneous presentation of malignant external otitis and temporal bone cancer.: Archives of Otolaryngology - Head and Neck Surgery. 617-23. 1993; 119: 687-9.

6. M. Arriaga, H. D. Curtin, H. Takahashi, and D. B. Kamerer, "The role of preoperative CT scans in staging external auditory meatus carcinoma: radiologic-pathologic correlation study, " Otolaryngology Head and Neck Surgery, vol. 105, no. 1, pp. 6- 11, 1991. 


\section{CASE REPORT}

7. G. Chee, P. Mok, and R. Sim, "Squamous cell carcinoma of the temporal bone: diagnosis, treatment and prognosis, " Singapore Medical Journal, vol. 41, no. 9, pp. 441-451, 2000.

8. Pensak ML, Geich LL, Gluckman JL, Shumrick KA. Laryngoscope, 1996, Oct;106: 1234-37.

9. Prabhu R, Hinerman RW, Indelicato DJ, et al: Squamous cell carcinoma of the external auditory canal: long-term clinical outcomes using surgery and external-beam radiotherapy. Am J Clin Oncol 32: 401-404, 2009.

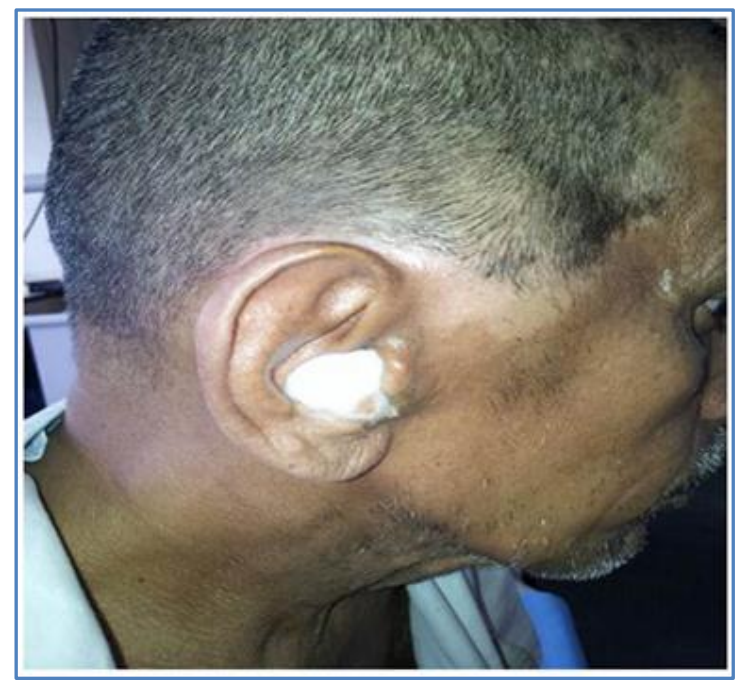

Fig. 1: Swelling in Pre-auricular and Post-auricular region

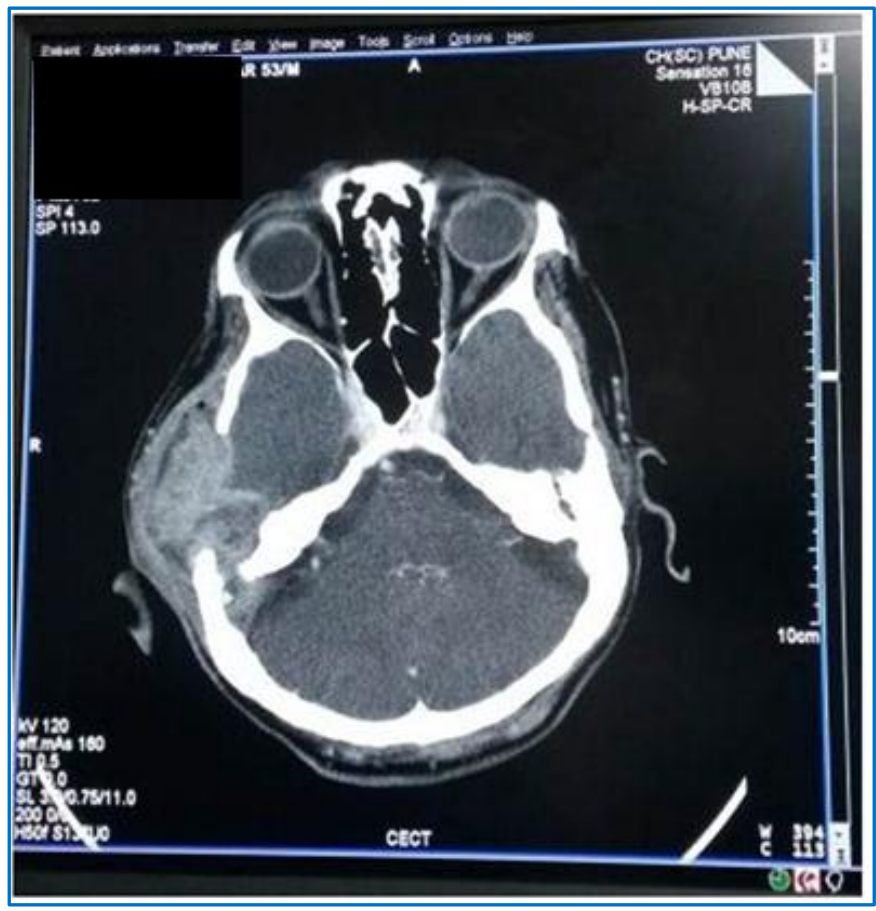

Fig. 2: CECT Brain showed revealed irregular heterogenoulsy enhancing lesion 


\section{CASE REPORT}

\section{AUTHORS: \\ 1. Tarun Sood \\ 2. Kaustubh Kahane \\ 3. Col S. K. Singh \\ 4. Suresh Mokamati}

\section{PARTICULARS OF CONTRIBUTORS:}

1. Clinical Tutor, Department of ENT, Armed Forces Medical College.

2. Resident, Department of ENT, Armed Forces Medical College.

3. Associate Professor, Department of ENT, Armed Forces Medical College.

FINANCIAL OR OTHER COMPETING INTERESTS: None
4. Resident, Department of ENT, Armed Forces Medical College.

\section{NAME ADDRESS EMAIL ID OF THE} CORRESPONDING AUTHOR:

\section{Dr. Kaustubh Kahane,}

Resident ENT,

Armed Forces Medical College,

Pune.

E-mail: drkaustubhkahane@gmail.com

Date of Submission: 17/11/2015.

Date of Peer Review: 21/11/2015.

Date of Acceptance: 11/05/2015.

Date of Publishing: 18/05/2015. 